\title{
New Insights into Wine Taste: Impact of Dietary Lipids on Sensory Perceptions of Grape Tannins
}

Ahmad Saad ${ }^{1}$, Julien Bousquet ${ }^{2}$, Nora Fernandez-Castro², Antoine Loquet ${ }^{1}$, Julie Géan ${ }^{1}$

1Univ. Bordeaux, CNRS, CBMN UMR 5348, IECB, F-33600 Pessac, France

Univ. Bordeaux, IUT Génie Biologique, F-24000 Périgueux, France<smiles>Oc1cc(O)c2c(c1)O[C@H](c1ccc(O)c(O)c1)[C@H](O)C2</smiles>

B

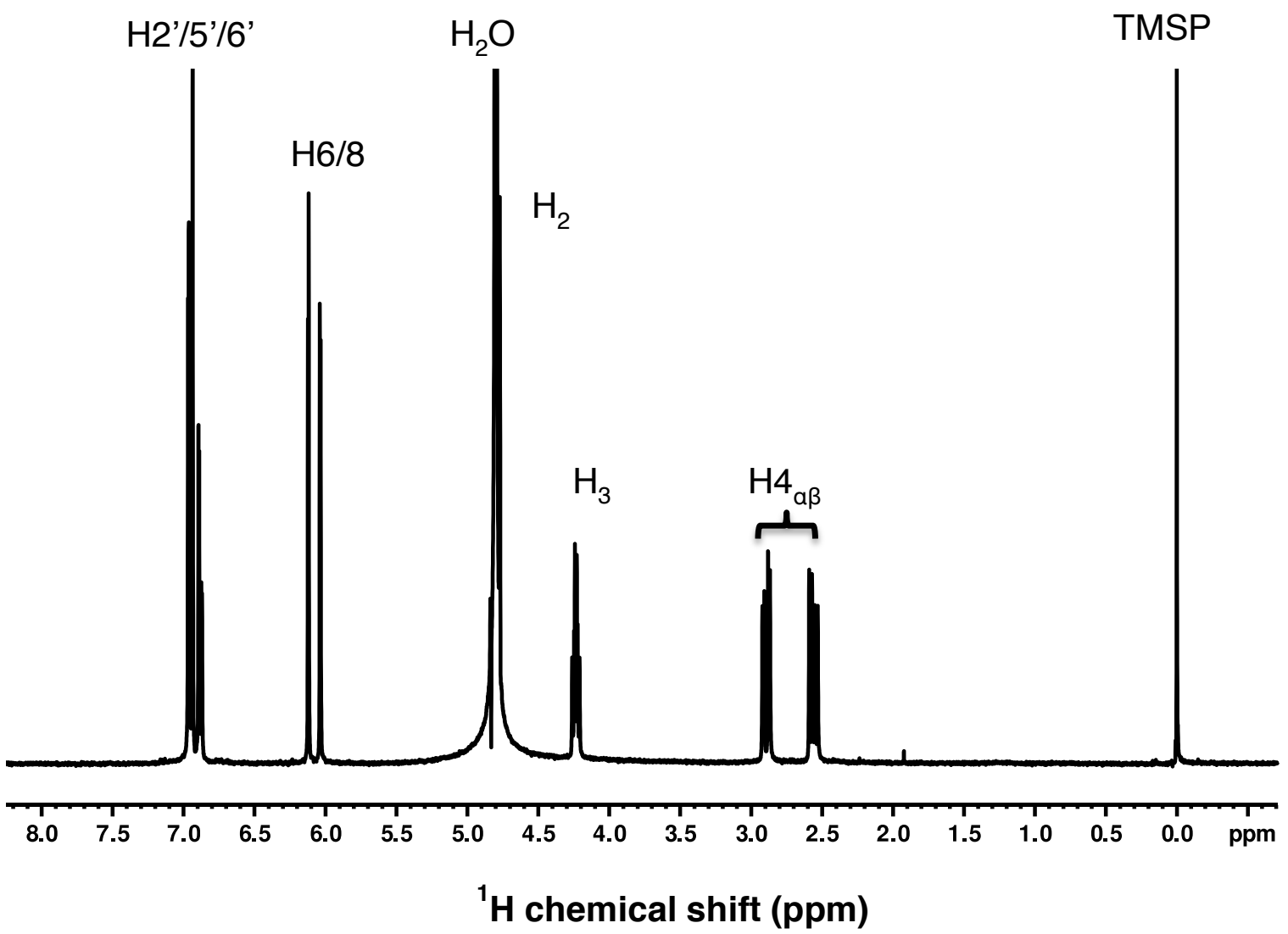

Figure S1. (A) Catechin chemical structure. (B) ${ }^{1} \mathrm{H}$ liquid state NMR spectrum of catechin in $\mathrm{H}_{2} \mathrm{O}$. The chemical shift reference (TMSP) was set to $0.0 \mathrm{ppm}$. Number of scans $=16,400 \mathrm{MHz}$ spectrometer, Temperature $25^{\circ} \mathrm{C}$. 


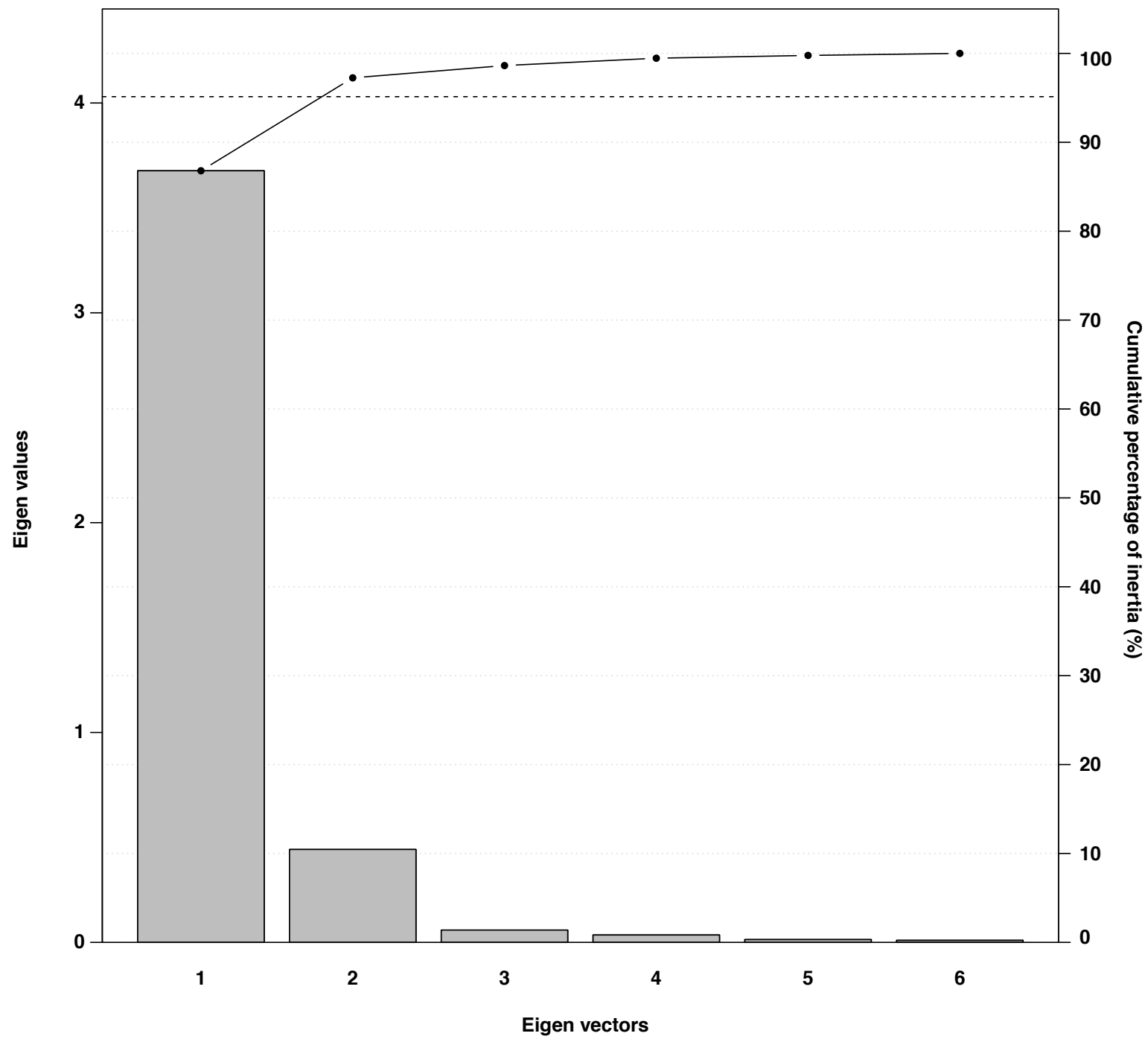

Figure S2. Inertia percent relating to the eigenvalues associated with the PCA eigenvectors. 
Table S1. Student's test scores for the oils tasted alone. The data corresponds to the p-value (above) and the statistical significance of the difference (below).

\begin{tabular}{|l|c|c|c|c|c|c|c|c|}
\hline & Intensity & Acidity & Fruity & Herbaceous & Dried fruit & Bitterness & Astringency & Persistence \\
\hline $\begin{array}{l}\text { Rapeseed vs } \\
\text { Grapeseed }\end{array}$ & 0.96 & 0.74 & 0.45 & 0.26 & 0.38 & 0.69 & 0.81 & 0.94 \\
NS & NS & NS & NS & NS & NS & NS & NS \\
\hline $\begin{array}{l}\text { Rapeseed vs } \\
\text { Olive }\end{array}$ & $\begin{array}{c}5.25 \mathrm{E}-27 \\
\star * *\end{array}$ & $\begin{array}{c}* .32 \mathrm{E}-09 \\
\star *\end{array}$ & $\begin{array}{c}7.10 \mathrm{E}-10 \\
\star *\end{array}$ & $\begin{array}{c}3.85 \mathrm{E}-04 \\
*\end{array}$ & $\begin{array}{c}6.24 \mathrm{E}-03 \\
*\end{array}$ & $\begin{array}{c}1.81 \mathrm{E}-11 \\
* *\end{array}$ & $\begin{array}{c}2.22 \mathrm{E}-03 \\
*\end{array}$ & $\begin{array}{c}6.30 \mathrm{E}-20 \\
* * *\end{array}$ \\
\hline $\begin{array}{l}\text { Olive vs } \\
\text { Grapeseed }\end{array}$ & $\begin{array}{c}1.47 \mathrm{E}-28 \\
\star * *\end{array}$ & $\begin{array}{c}* .24 \mathrm{E}-09 \\
\star *\end{array}$ & $\begin{array}{c}2.24 \mathrm{E}-08 \\
\star *\end{array}$ & $\begin{array}{c}1.84 \mathrm{E}-02 \\
*\end{array}$ & $\begin{array}{c}0.07 \\
\text { NS }\end{array}$ & $\begin{array}{c}3.09 \mathrm{E}-12 \\
* *\end{array}$ & $\begin{array}{c}3.57 \mathrm{E}-03 \\
*\end{array}$ & $\begin{array}{c}8.94 \mathrm{E}-20 \\
* * *\end{array}$ \\
\hline
\end{tabular}


Table S2. Student's test scores for the tannins tasted alone. The data corresponds to the p-value (above) and the statistical significance of the difference (below).

\begin{tabular}{|l|c|c|c|c|c|c|}
\hline & Intensity & Acidity & Fruity & Bitterness & Astringency & Persistence \\
\hline A0.15 vs & $1.82 \mathrm{E}-06$ & 0.08 & 0.07 & $3.55 \mathrm{E}-05$ & $2.60 \mathrm{E}-06$ & $5.10 \mathrm{E}-04$ \\
A1.16 & $* *$ & NS & NS & $* *$ & $*$ \\
\hline GS0.15 vs & $7.14 \mathrm{E}-12$ & $5.85 \mathrm{E}-04$ & 0.10 & $4.35 \mathrm{E}-05$ & $2.43 \mathrm{E}-08$ & $2.71 \mathrm{E}-06$ \\
GS1.16 & $* *$ & $*$ & NS & $*$ & $* *$ \\
\hline A0.15 vs & $8.93 \mathrm{E}-03$ & 0.56 & 0.86 & 0.65 & 0.48 & 0.79 \\
GS0.15 & $*$ & NS & NS & NS & NS & NS \\
\hline A1.16 vs & $1.80 \mathrm{E}-02$ & 0.69 & 0.56 & 0.13 & 0.07 & 0.52 \\
GS1.16 & $*$ & NS & NS & NS & NS & NS \\
\hline
\end{tabular}


Table S3. Inertia and cumulative inertia percentages for the six eigenvectors (EV).

\begin{tabular}{lcccccc}
\hline & EV 1 & EV 2 & EV 3 & EV 4 & EV 5 & EV 6 \\
\hline Inertia \% & 86.78 & 10.47 & 1.38 & 0.83 & 0.31 & 0.23 \\
Cumulated inertia \% & 86.78 & 97.25 & 98.63 & 99.46 & 99.77 & 100 \\
\hline
\end{tabular}


Table S4. Student's test scores for the tannins tasted alone and after the ingestion of oils. The data corresponds to the p-value (above) and the statistical significance of the difference (below).

\begin{tabular}{|l|c|c|c|c|c|c|}
\hline & Intensity & Acidity & Fruity & Bitterness & Astringency & Persistence \\
\hline A0.15 vs & 0.55 & 0.85 & 0.06 & 0.81 & 0.87 & 0.59 \\
Rapeseed & NS & NS & NS & NS & NS & NS \\
\hline A0.15 vs & $1.42 \mathrm{E}-03$ & $9.74 \mathrm{E}-03$ & $6.08 \mathrm{E}-06$ & $2.89 \mathrm{E}-02$ & 0.88 & $2.01 \mathrm{E}-05$ \\
Olive & $* *$ & $* *$ & $* *$ & $*$ & NS & $* * *$ \\
\hline A0.15 vs & 0.06 & 0.59 & $2.74 \mathrm{E}-02$ & 0.23 & 0.62 & 0.27 \\
Grapeseed & NS & NS & $*$ & NS & NS & NS \\
\hline A1.16 vs & 0.55 & 0.70 & 0.18 & 0.41 & 0.07 & 0.26 \\
Rapeseed & NS & NS & NS & NS & NS & NS \\
\hline A1.16 vs & 0.41 & $2.89 \mathrm{E}-02$ & $3.25 \mathrm{E}-02$ & 0.37 & 0.12 & 0.13 \\
Olive & NS & $*$ & $*$ & NS & NS & NS \\
\hline A1.16 vs & 0.42 & 0.32 & 0.80 & 0.51 & $3.85 \mathrm{E}-03$ & 0.61 \\
Grapeseed & NS & NS & NS & NS & $* *$ & NS \\
\hline PR0.15 vs & 0.15 & 0.91 & 0.13 & 0.55 & 0.00 & 0.49 \\
Rapeseed & NS & NS & NS & NS & NS & NS \\
\hline PR0.15 vs & $2.94 \mathrm{E}-07$ & $5.70 \mathrm{E}-03$ & $1.65 \mathrm{E}-05$ & 0.12 & 0.21 & $1.72 \mathrm{E}-04$ \\
Olive & $* * *$ & $* *$ & $* * *$ & NS & NS & $* * *$ \\
\hline PR0.15 vs & $9.06 \mathrm{E}-04$ & 0.44 & $5.26 \mathrm{E}-03$ & 0.93 & $2.57 \mathrm{E}-02$ & 0.13 \\
Grapeseed & $* * *$ & NS & $* *$ & NS & $*$ & NS \\
\hline PR1.16 vs & 0.43 & 0.59 & 0.95 & 0.65 & 0.32 & 0.77 \\
Rapeseed & NS & NS & NS & NS & NS & NS \\
\hline PR1.16 vs & $1.14 \mathrm{E}-02$ & 0.36 & $9.56 \mathrm{E}-04$ & 0.16 & 0.31 & $1.48 \mathrm{E}-02$ \\
Olive & $*$ & NS & $* * *$ & NS & NS & $*$ \\
\hline PR1.16 vs & 0.94 & 0.48 & 0.61 & 0.98 & $1.95 \mathrm{E}-02$ & 0.70 \\
Grapeseed & NS & NS & NS & NS & $*$ & NS \\
\hline
\end{tabular}

